\section{Measurements of the Neutron Polarized Structure Function at SLAC}

\section{CHARLES C. YOUNG}

Stanford Linear Accelerator Center Stanford University, Stanford, CA 94309 representing the E-142 Collaboration ${ }^{+}$

\section{ABSTRACT}

Detailed measurements of unpolarized or spin-averaged nucleon structure functions over the past two decades have led to detailed knowledge of the nucleon's internal momentum distribution. Polarized nucleon structure function measurements, which probe the nucleon's internal spin distribution, started at SLAC in 1976. E-142 has recently measured the neutron polarized structure function $g_{1}^{n}(x)$ over the range $0.03<\mathrm{x}<0.6$ at an average $\mathrm{Q}^{2}$ of $2 \mathrm{GeV}^{2}$ and found the integral $I^{n}=\int_{0}^{1} g_{1}^{n}(x) d x=-0.022 \pm 0.011$. E-143, which took data recently, has measured $g_{1}^{p}$ and $g_{1}^{d}$. Two more experiments (E-154 and E-155) will extend these measurements to lower $x$ and higher $Q^{2}$.

Presented at the International Workshop on Deep Inelastic Scattering and Related Subjects, Eilat, Israel, February 6-11, 1994

\footnotetext{
- Work supported by Department of Energy contract DE-AC03-76SF00515.

+ P.L. Anthony, R.G. Amold, H.R. Band, H. Borel, P.E. Bosted, V. Breton, G.D. Cates, T.E. Chupp, F.S. Dietrich, J. Dunne, R. Erbacher, J. Fellbaum, H. Fonvieille, R. Gearhart, R. Holmes, E.W. Hughes, J.R. Johnson, D. Kawall, C. Keppel, S.E. Kuhn, R.M. Lombard-Nelsen, J. Marroncle, T. Maruyama, W. Meyer, Z.-E. Meziani, H. Middleton, J. Morgenstern, N.R. Newbury, G.G. Petratos, R. Pitthan, R. Prepost, Y. Roblin, S.E. Rock, S.H. Rokni, G. Shapiro, T. Smith, P.A. Souder, M. Spengos, F. Staley, L.M. Stuart, Z.M. Szalata, Y. Terrien, A.K. Thompson, J.L. White, M. Woods, J. Xu, C.C. Young, G. Zapalac
}

\section{Theoretical Background}

\subsection{Polarized Structure Functions $g_{1}(x)$ and $g_{2}(x)$}

The differential cross section for scattering a polarized electron from a polarized nucleon is characterized by the formula:

$$
\frac{d^{2} \sigma^{\uparrow \downarrow}}{d Q^{2} d v}-\frac{d^{2} \sigma^{\uparrow \uparrow}}{d Q^{2} d v}=\frac{4 \pi \alpha^{2}}{Q^{2} E^{2}}\left[M\left(E+E^{\prime} \cos \theta\right) G_{1}\left(Q^{2}, v\right)-Q^{2} G_{2}\left(Q^{2}, v\right)\right]
$$

where $\uparrow \downarrow(\uparrow \uparrow)$ indicates longitudinal target spin antiparallel (parallel) to the incident electron spin. The incident electron beam energy is $\mathrm{E}, \mathrm{E}^{\prime}$ is the scattered electron energy, and $\theta$ is the electron scattering angle. The mass of the nucleon is $M, v=(E-E)$ is the energy loss of the electron, $-\mathrm{Q}^{2}$ is the square of the four momentum of the virtual photon, and $\alpha$ is the fine structure constant. In the scaling limit, the functions $G_{1}\left(Q^{2}, v\right)$ and $\mathrm{G}_{2}\left(\mathrm{Q}^{2}, v\right)$ can be written in terms of the polarized structure functions $g_{1}(x)$ and $g_{2}(x)$ :

$$
\begin{aligned}
& g_{1}(x)=M^{2} v G_{1}\left(Q^{2}, v\right), \\
& g_{2}(x)=M v^{2} G_{2}\left(Q^{2}, v\right),
\end{aligned}
$$

where $x=Q^{2} /(2 M v)$ is the scaling variable. It can be shown that $g_{1}$ is given by

$$
g_{1}(x)=\frac{F_{2}\left(x, Q^{2}\right)}{2 x(1+R)}\left[A_{1}\left(x, Q^{2}\right)+\gamma A_{2}\left(x, Q^{2}\right)\right],
$$

where $F_{2}$ is the unpolarized structure function, $A_{1}$ is the cross-section asymmetry for fully polarized virtual photon on logitudinally polarized nucleons, $\gamma$ is a kinematic factor given by $\gamma^{2}=\left(4 \mathrm{M}^{2} \mathrm{X}^{2}\right) / \mathrm{Q}^{2}, \mathrm{~A}_{2}$ is a similar cross section asymmetry for transversely polarized nucleon, and $\mathrm{R}$ is the ratio of longitudinally to transversely polarized photon cross-sections $\left(\sigma_{L} / \sigma_{T}\right)$.Virtual photon asymmetries $\mathrm{A}_{1}$ and $\mathrm{A}_{2}$ are related to the corresponding cross section asymmetry for electrons $A_{y}\left(x, Q^{2}\right)$ and $A_{\perp}\left(x, Q^{2}\right)$ by

$$
\begin{aligned}
& A_{11}=D\left(A_{1}+\eta A_{2}\right), \\
& A_{\perp}=d\left(A_{2}-\zeta A_{1}\right),
\end{aligned}
$$

where $\|(L)$ refers to target nucleon spin aligned along (transverse to) the electron beam spin direction. The kinematic factor $\mathrm{D}$ relating electron polarization to that of the virtual photon is given by

$$
D=\frac{1-\varepsilon\left(\frac{E^{\prime}}{E}\right)}{1+\varepsilon R}
$$

Note that $\varepsilon^{-1}=1+2\left(1+\frac{v^{2}}{Q^{2}}\right) \tan ^{2}\left(\frac{\theta}{2}\right), \eta=\left(\varepsilon \sqrt{Q^{2}}\right) /(E-E \varepsilon), d=D \sqrt{(2 \varepsilon) /(1+\varepsilon)}$ and $\zeta=\eta(1+\varepsilon) / \varepsilon$. Thus, measurements of $A_{\|}$and $A_{\perp}$ give $A_{1}$ and $A_{2}$, and hence will allow a determination of $g_{1}$. The kinematic factors ensure that $g_{1}$ is insensitive to measurement errors on $A_{1}$. Since $\zeta$ is small, $A_{\perp}$ depends primarily on $A_{2}$, which is limited by unitarity 
considerations to $\left|A_{2}\right| \leq \sqrt{R}$. Since $\mathrm{R}$ is known to be small, and $\gamma$ can also be shown to be small for the kinematics of this experiment, the second term in $g_{1}$ is relatively unimportant.

The behavior of $\mathrm{g}_{1}$ near $\mathrm{x}=0$ and $\mathrm{x}=1$ is known. As $x \rightarrow 1, \mathrm{~g}_{1}$ must go to zero as $F_{2} \rightarrow 0$ since the other factors are finite. Regge theory is reliable at low $\mathrm{x}$ and predicts that $g_{1}(x) \propto x^{\alpha}$ as $x \rightarrow 0$.

The equation for $g_{1}(x)$ can be easily understood within the quark parton model (QPM). It is interpreted as the difference distribution between quarks whose spin is parallel and antiparallel to the nucleon's spin.

$$
g_{1}(x)=\frac{1}{2} \sum e_{i}^{2}\left(q_{i}^{\dagger}(x)-q_{i}^{\downarrow}(x)\right)
$$

Similarly, the unpolarized structure function $F_{1}$ is interpreted as the sum.

$$
F_{1}(x)=\frac{1}{2} \sum e_{i}^{2}\left(q_{i}^{\dagger}(x)+q_{i}^{\downarrow}(x)\right)
$$

The arrow $\tau(\downarrow)$ indicates quark spin parallel(antiparallel) to nucleon spin. It can be seen that the ratio of these two equations together with the well known relationship between $F_{1}$ and $\mathrm{F}_{2}$ yields the leading term in $\mathrm{g}_{1}$ above.

\subsection{Sum Rules}

While there are no rigorous predictions of the detailed functional forms of $g_{1}^{p}(\mathrm{x})$ and $g_{1}^{n}(\mathrm{x})$, Bjorken derived a fundamental sum rule on their integrals. ${ }^{1}$ Denoting by IP the integral $\int_{0}^{1} g_{1}^{p}(x) d x$ for a proton and $\mathrm{I}^{\mathrm{n}}$ the integral $\int_{0}^{1} g_{1}^{n}(x) d x$ for a neutron, the Bjorken sum rule is given by:

$$
I^{p}-I^{n}=\frac{1}{6}\left(\frac{g_{A}}{g_{V}}\right)\left[1+O\left(\alpha_{s}\left(Q^{2}\right)\right)\right]
$$

where $g_{\mathrm{A}}$ and $g_{\mathrm{V}}$ are the axial and vector coupling constants measured very accurately in nucleon $\beta$ decays. $Q C D$ corrections are given to first order by $-\left(\alpha_{s} / \pi\right) .{ }^{2}$ Violations of the Bjorken sum rule would seriously undermine the validity of QCD.

Independent sum rules for IP and $\mathrm{I}^{\mathrm{n}}$ have been derived by Ellis and Jaffe: ${ }^{3}$

$$
\begin{aligned}
& I^{p}=\frac{1}{18}(9 F-D)\left[1+O\left(\alpha_{s}\left(Q^{2}\right)\right)\right] \\
& I^{n}=\frac{1}{18}(6 F-4 D)\left[1+O\left(\alpha_{s}\left(Q^{2}\right)\right)\right]
\end{aligned}
$$

where $\mathrm{F}$ and $\mathrm{D}$ are coupling constants measured in hyperon decay. ${ }^{4}$ The derivation relies upon SU(3) flavor symmetry and the assumption of an unpolarized strange sea. Thus, violations of the Ellis-Jaffe sum rules need not pose a fundamental problem for QCD.

1 J.D. Bjorken, Phys. Rev. 148 (1966) 1467, Phys. Rev. D1 (1970) 1376.

2 Higher order corrections have also been calculated. See S.A. Larin and J.A.M. Vermascren, Phys. Lett. B259 (1991) 345 .

3 J. Ellis and R.L. Jaffe, Phys. Rev D9 (1974) 1444. See S.A. Larin, Phys. Lett. B334 (1994) 192, and A. L. Kataev, Report No. CERN-TH- 7427/94 for QCD corrections.

${ }^{4}$ R.L. Jaffe and A.V. Manohar, Nucl. Phys. B337 (1990) 509, F.E. Close and R.G. Roberts, Phys. Lett. B316 (1993) 165 .

\subsection{Quark Spin Contribution}

The total quark contribution to nucleon spin, $\Delta \mathrm{q}$, is the sum of the individual quark flavor contributions $\Delta \mathrm{u}, \Delta \mathrm{d}$, and $\Delta s$.

$$
\Delta u=\int_{0}^{1}\left[u^{\uparrow}(x)-u^{\downarrow}(x)\right] d x,
$$

where $T(\downarrow)$ indicates quark spin parallel (antiparallel) to nucleon spin. In QPM, these quantities are related to experimental measurements of hyperon decay constants, beta decay measurements, ${ }^{5}$ and polarized structure functions:

$$
\begin{aligned}
D-F & =\Delta s-\Delta d \approx 0.339 \pm 0.011, \\
\frac{g_{A}}{g_{V}} & =\Delta u-\Delta d \approx 1.257 \pm 0.003, \\
I^{p} & =\frac{1}{2}\left[\frac{4}{9} \Delta u+\frac{1}{9} \Delta d+\frac{1}{9} \Delta s\right], \\
I^{n} & =\frac{1}{2}\left[\frac{1}{9} \Delta u+\frac{4}{9} \Delta d+\frac{1}{9} \Delta s\right],
\end{aligned}
$$

By combining the first two equations with a measurement of either $\mathrm{IP}$ or $\mathrm{I}^{\mathrm{n}}$, the individual quark spin contributions can be calculated.

SLAC Experiments E- $80^{6}$ and E-1307 made the first measurements of polarized proton structure function. They were followed by the European Muon Collaboration group (EMC). The latter's measurement ${ }^{8}$ of $I^{p}=0.126 \pm 0.018$ implies

$$
\begin{gathered}
\Delta u=0.74 \pm 0.05, \\
\Delta d=-0.52 \pm 0.05, \\
\Delta s=-0.18 \pm 0.06,
\end{gathered}
$$

$\Delta q=\Delta u+\Delta d+\Delta s=0.05 \pm 0.16$.

This rather surprising conclusion that quarks carry little of the spin of the proton has been dubbed the Spin Crisis. The results also imply that there is substantial strange sea polarization. There has been much theoretical speculation on this. ${ }^{9}$

\section{SLAC Experiment E-142}

The goal of SLAC experiment E-14210 is to perform a high statistics measurement of the neutron's polarized structure function $g_{1}^{n}$ and hence obtain $I^{n}$. This can be combined with a

5 Review of Particle Properties, Phys. Rev. D45 (1992) 1.

${ }^{6}$ M.J. Alguard et al., Phys. Rev. Lett. 37 (1976) 1258 and M.J. Alguard et al., Phys. Rev. Lett. 37 (1976) 1261.

7 G. Baum et al., Phys. Rev. Lett. 51 (1083) 1135.

8 J. Ashman et al., Phys. Lett. B206 (1988) 364 and J. Ashman et al., Nucl. Phys. B328 (1989) 1.

${ }^{9}$ G. Altarelli et al., Phys. Lett. B212 (1988) 391, R.D. Carlitz et al., Phys. Lett. B214 (1988) 229, S. Brodsky et al., Phys. Lett. B206 (1988) 309, J. Ellis et al., Phys. Lett. B213 (1988) 73.

10 P.L. Anthony et al.., Phys. Rev. Lett. 71 (1993) 959. 


\section{DISCLAIMER}

Portions of this document may be illegible in electronic image products. Images are produced from the best available original document. 


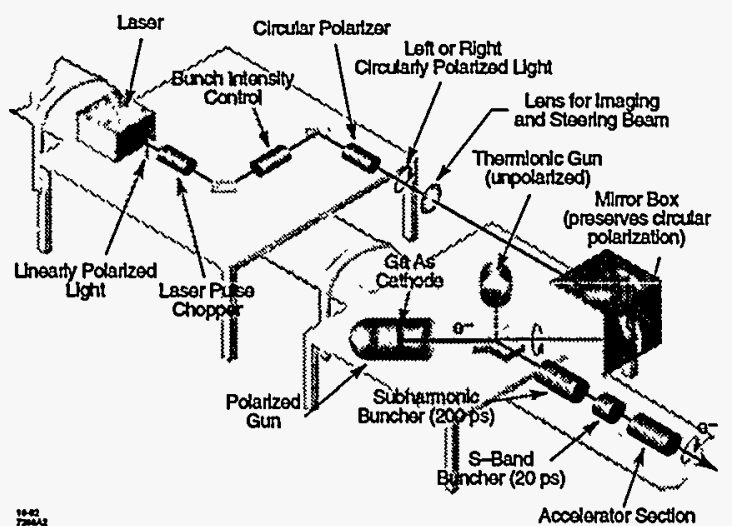

Figure 1: Schematic of the SLAC polarized electron source.

measurement of IP to check the Bjorken sum rule. It can also be used independently of IP to calculate quark contribution to nucleon spin.

\subsection{Beam}

Circularly polarized light impinges on an AlGaAs cathode11 as shown in Fig. 1 . Conservation of angular momentum restricts ionization from certain energy-degenerate spin states, and their relative populations give rise to a net electron beam polarization $\mathrm{P}_{\mathrm{b}}$ of typically $39 \%$ in this experiment. See Table I for typical beam parameters. Beam polarization was measured with Møller scattering, and was found to be stable over the entire run. Polarization sign was chosen randomly on a pulse by pulse basis; thus, false asymmetries due to changes in detector acceptance or response are minimized

Table I: Beam parameters for E-142.

\begin{tabular}{|l|l|}
\hline Energy, $\mathrm{E}$ & 19,22, and $26 \mathrm{GeV}$ \\
\hline Intensity & $0.5-2 \times 10^{11}$ electrons per pulse \\
\hline Pulse duration & $0.8-1.4 \mu \mathrm{sec}$ \\
\hline Polarization, $\mathrm{P}_{\mathrm{b}}$ & $38.8 \pm 1.6 \%$ \\
\hline Polarization Reversal & random, pulse by pulse \\
\hline Repetition Rate & $120 \mathrm{~Hz}$ \\
\hline
\end{tabular}

\subsection{Target}

The target uses ${ }^{3} \mathrm{He}$, which is polarized through spin exchange collisions with optically pumped polarized rubidium vapor. ${ }^{12} \mathrm{~A}$ two-chamber design was used. ${ }^{13}$ Figure 2 shows the upper pumping chamber pumped by five high powered laser systems producing up to

11 T.E. Chupp et al.1, Phys. Rev. C45 (1992) 915.

12 T. Maruyama et al.., J. Appl. Phys. 73 (1993) 5189.

13 T.E. Chupp et al., Phys. Rev. C36 (1987) 2244.

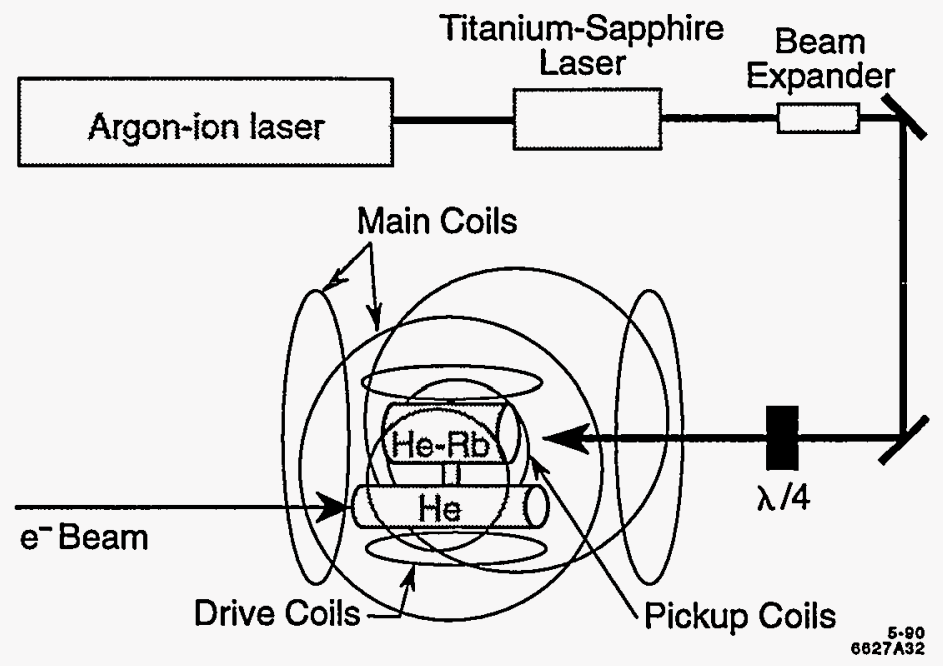

Figure 2: Schematic layout of the polarized ${ }^{3} \mathrm{He}$ target. Five sets of lasers optically pump rubidium vapor in the top chamber, and ${ }^{3} \mathrm{He}$ nuclei acquire polarization through spin exchange collisions. Incident electrons scatter off nuclei in the bottom chamber. Two sets of Helmholtz coils hold the target spin in either longitudinal or transverse directionss. Drive and pickup coils are used to measured polariztion.

$20 \mathrm{~W}$ of infrared laser light. The lower target chamber has a length of $30 \mathrm{~cm}$ with 0.012 cm-thick glass end windows. It was filled with $2.3 \times 10^{20}{ }^{3} \mathrm{He}$ atoms/cc. The ${ }^{3} \mathrm{He}$ target polarization $P_{t}$ was measured using nuclear magnetic resonance (NMR) techniques. A precision of $\Delta \mathrm{P}_{\mathrm{t}} / \mathrm{P}_{\mathrm{t}}=7 \%$ was achieved, $\mathrm{P}_{\mathrm{t}}$ varying slowly between $30 \%$ and $40 \%$ during the experiment, and its direction reversed frequently. Data were taken with target polarization along and transverse to beam direction to measure both $A_{\|}$and $A_{\perp}$.

The exclusion principle ensures that the two proton spins are antiparallel in the ground state ${ }^{3} \mathrm{He}$ wave function. Protons do not contribute to measured asymmetry. ${ }^{14}$ This contrasts with a deuteron target for which the proton's relatively large asymmetry must be subtracted statistically.

\subsection{Spectrometer}

Scattered electrons are detected in two independently operating spectrometers (see Fig. 3)..$^{15}$ The scattering angles are $4.5^{\circ}$ and $7^{\circ}$, respectively, measuring overlapping ranges of $x$ from 0.03 to 0.6 with $Q^{2}$ greater than $\approx 1 \mathrm{GeV}^{2}$. Approximately $4 \times 10^{8}$ events were collected.

Magnetic deflection and scintillation hodoscopes measure momentum with a precision of $\sim 2 \%$. The 200-element 24-radiation-length lead glass array measures electron energy with a

14 Higher order corrections are $\sim 10 \pm 2 \%$. See for example R.M. Woloshin, Nucl. Phys. 496A (1989) 749, and Ciofi degli Atti et al., Univ. of Perugia Preprint 75/93.

15 G.G. Petratos et al., SLAC-PUB-5678 (1991). 


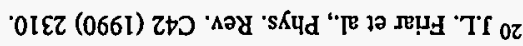
8ES (\$86I) $6 Z \mathrm{~J}^{\circ}$ '

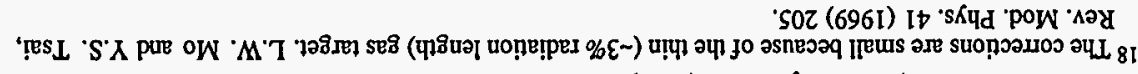

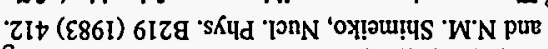

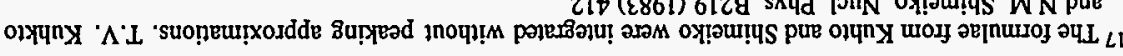

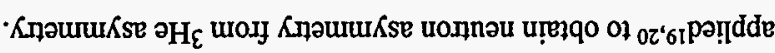

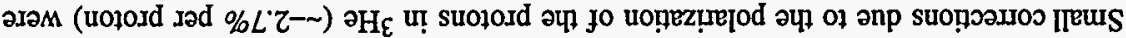

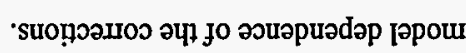

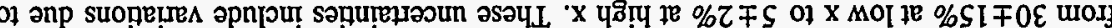

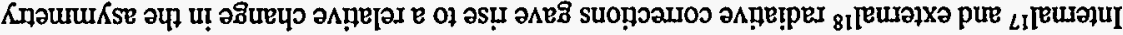
oIaz y!l M

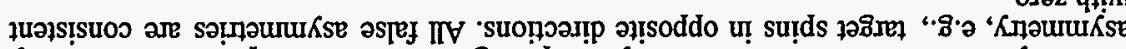

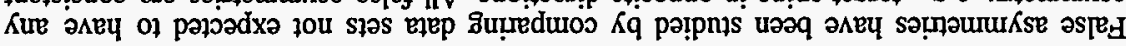

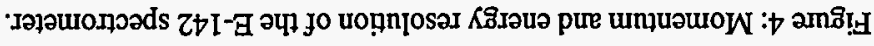

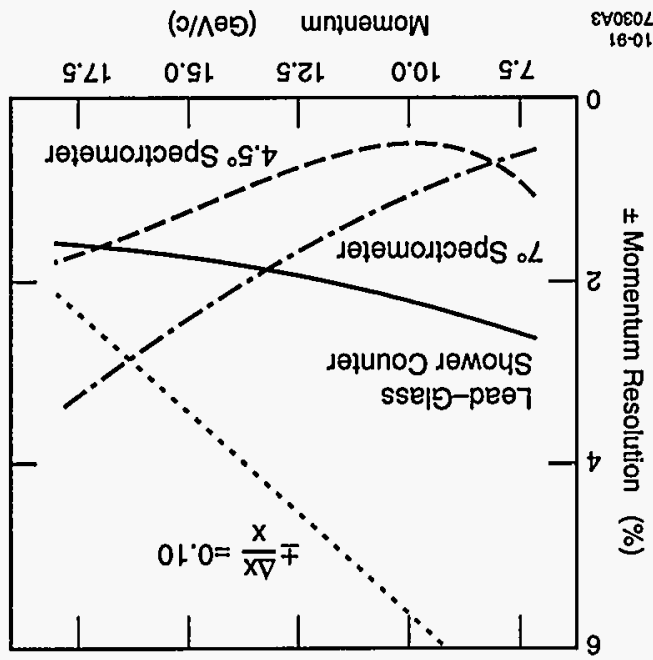

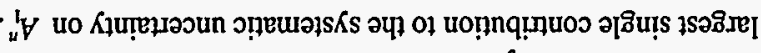

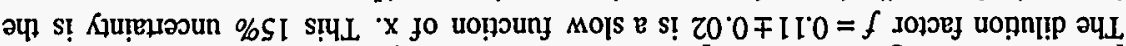
'sse

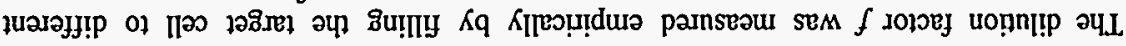

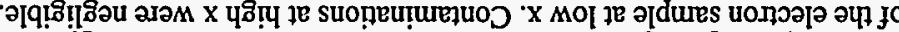

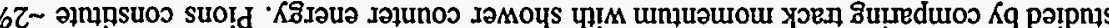

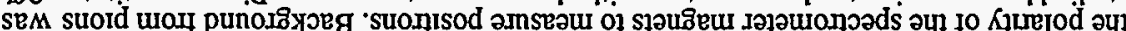

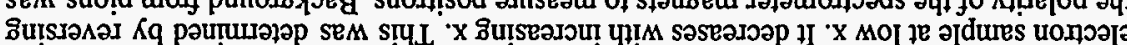

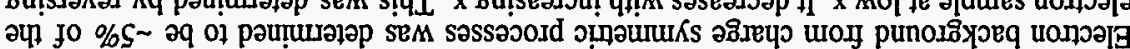

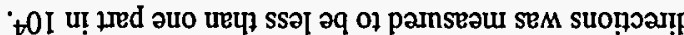

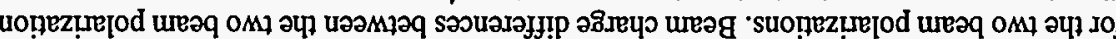

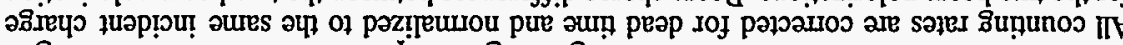

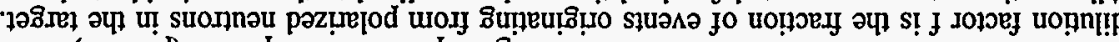

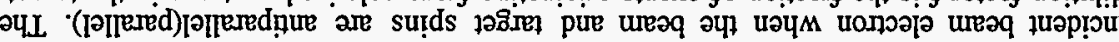

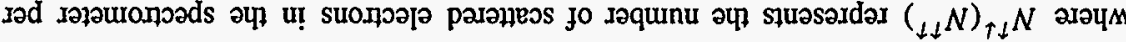

$$
f^{\prime} d^{q} d^{\prime \prime} \forall=\frac{\left({ }_{\downarrow \downarrow} N+{ }_{\uparrow \downarrow} N\right)}{\left({ }_{\downarrow \downarrow} N-{ }_{\uparrow \downarrow} N\right)}=\nabla
$$

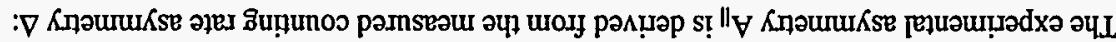

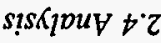

ưnน⿰冫⿱宀⿻三丨口

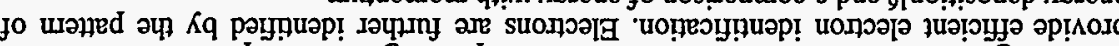

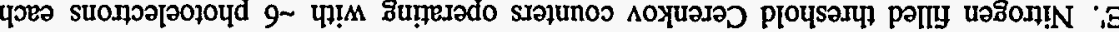

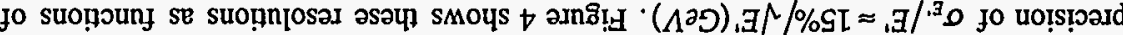

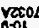

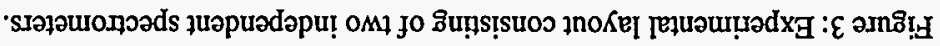
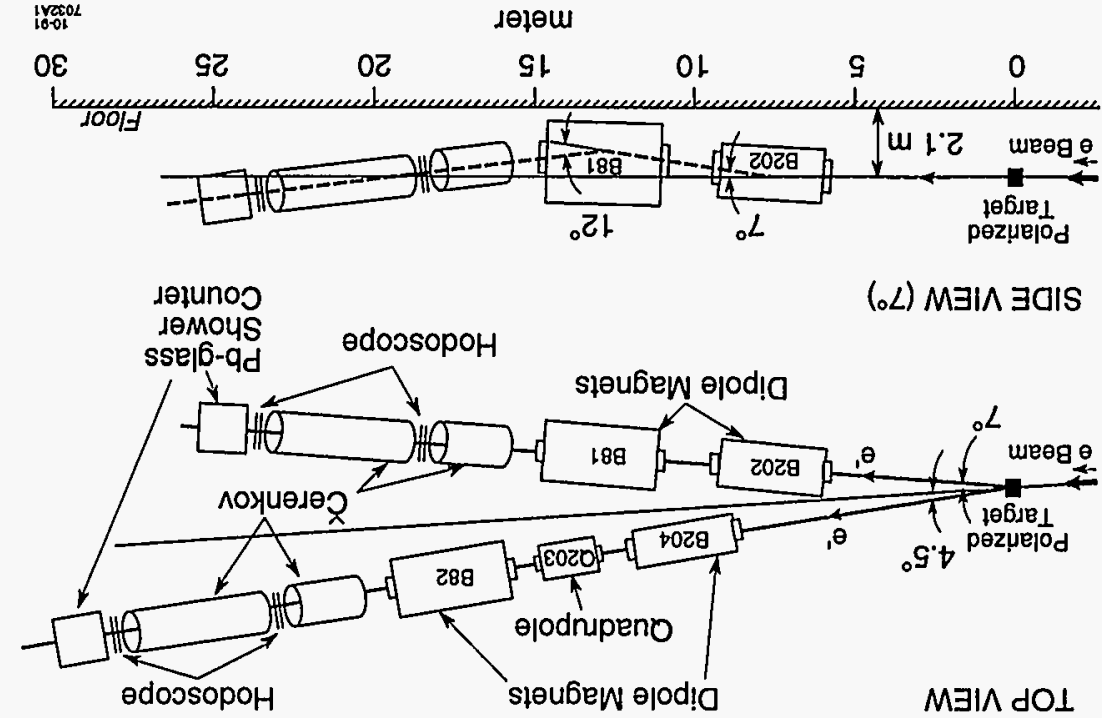


\subsection{Results}

Figure 5 shows $A_{1}^{n}$ as a function of $\mathrm{Q}^{2}$ for different x ranges. Results are consistent with being $Q^{2}$-independent over the measured range. ${ }^{21}$ Data over all $Q^{2}$ will therefore be averaged when studying $x$ dependence. Measured neutron asymmetries and structure functions are given in Table II. Figure 6 shows $A_{1}^{n}$ and $g_{1}^{n}$ with statistical and systematic uncertainties added in quadrature as functions of $x$. They are the most precise measurements at this time.22

The integral of $g_{1}^{n}(x)$ over the measured $x$ range is therefore $-0.019 \pm 0.007$ (stat) \pm 0.006 (sys) at an average $\mathrm{Q}^{2}$ of $2 \mathrm{GeV}^{2}$. Measurements of $A_{1}^{n}(x)$ at different values of $\mathrm{x}$ have different average $\mathrm{Q}^{2}$. Using the measured $\mathrm{Q}^{2}$ dependence of unpolarized structure functions, they can be corrected to a common $\mathrm{Q}^{2}$ value of $2 \mathrm{GeV}^{2}$ before integration over $\mathrm{x}$. The integral result remains unchanged. Systematic uncertainties on $\mathrm{I}^{\mathrm{n}}$ are summarized in Table 3.

Table II: Neutron asymmetry results and structure functions from E-142. The first error is statistical and the second systematic.

\begin{tabular}{|c|c|c|c|c|c|}
\hline$\langle\mathrm{x}\rangle$ & $\left\langle\mathrm{Q}^{2}\right\rangle$ & $A_{1}^{n}$ & \multicolumn{1}{|c|}{$\Delta A_{1}^{n}$} & $g_{1}^{n}$ & $\Delta g_{1}^{n}$ \\
\hline 0.025 & 0.96 & 0.066 & $.109 / .019$ & 0.267 & $.446 / .100$ \\
\hline 0.035 & 1.1 & -0.058 & $.056 / .021$ & -0.175 & $.169 / .052$ \\
\hline 0.050 & 1.3 & -0.095 & .05 & -0.228 & $.079 / .061$ \\
\hline 0.078 & 1.6 & -0.062 & $.031 / .031$ & -0.095 & $.048 / .026$ \\
\hline 0.124 & 2.3 & -0.136 & $.030 / .038$ & -0.133 & $.029 / .031$ \\
\hline 0.175 & 2.7 & -0.087 & $.041 / .037$ & -0.057 & .0271 .014 \\
\hline 0.248 & 3.1 & -0.020 & $.046 / .055$ & -0.008 & .0191 .006 \\
\hline 0.344 & 3.4 & 0.029 & $.091 / .068$ & 0.006 & $.020 / .003$ \\
\hline 0.466 & 5.2 & 0.030 & $.219 / .100$ & 0.003 & $.024 / .002$ \\
\hline
\end{tabular}

21 The SMC collaboration has combined their deuteron results with proton results from SLAC E-80, E130 , and the EMC collaboration at Cern to derive $A_{1}$ for the neutron. These results extend to $Q^{2} \sim 25$ $\mathrm{GeV}^{2}$, and to within their much larger uncertainties do not observe any $Q^{2}$ dependence compared with the data reported here. See B. Adeva et al, Cem Preprint CERN-PPE/93-206.

22 B. Adeva et al., Phys. Lett. B302 (1993) 533; and K. Abe et al., SLAC-PUB-6734.
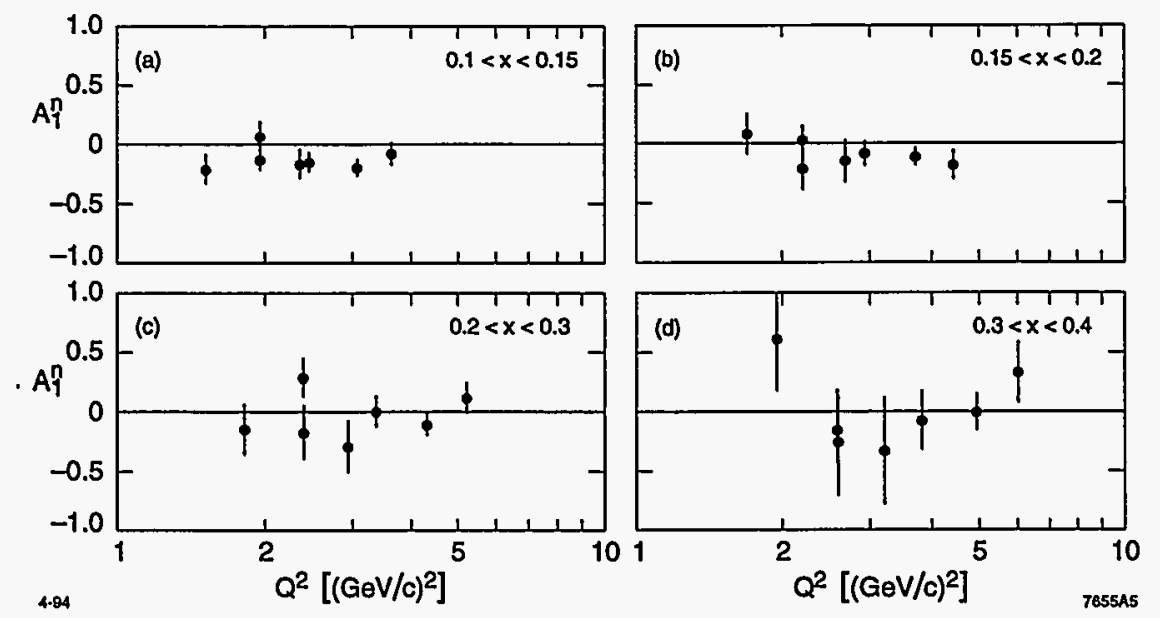

Figure 5: $A_{1}^{n}$ vs $\mathrm{Q}^{2}$ for four ranges of $\mathrm{x}$. Results are independent of $\mathrm{Q}^{2}$. Supply topdraw files.
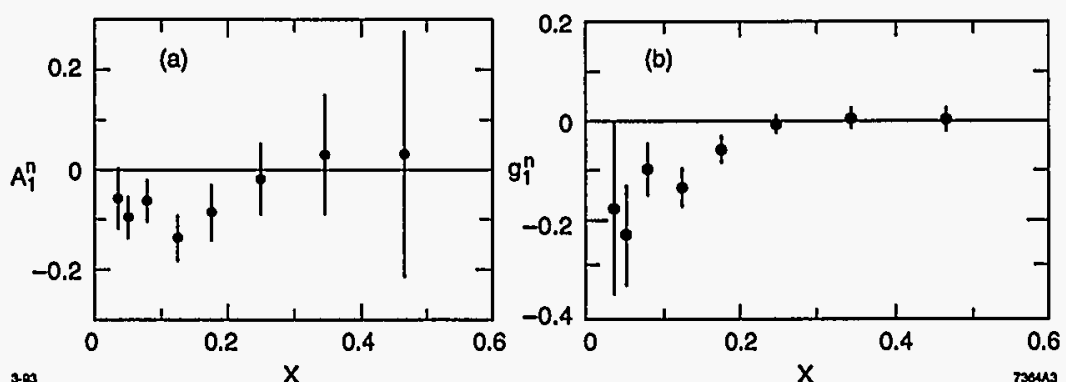

Figure 6: Results for neutron asymmetries $A_{1}^{n}$ and the neutron spin structure function $\mathrm{g}_{1}{ }^{\mathrm{n}}$ as functions of $x$ averaged over $Q^{2}$. Statistical and systematic errors are added in quadrature.

Table III: Summary of systematic uncertainties and their effects on In.

\begin{tabular}{|l|l|}
\hline Dilution Factor, $\mathrm{f}$ & 0.003 \\
\hline $\mathrm{A}_{2}$ & 0.003 \\
\hline $\mathrm{F}_{2}$ & 0.002 \\
\hline Target polarization, $\mathrm{P}_{\mathrm{t}}$ & 0.002 \\
\hline Beam polarization, $\mathrm{P}_{\mathrm{b}}$ & 0.001 \\
\hline Radiative correction & 0.001 \\
\hline $\mathrm{R}$ & 0.001 \\
\hline Total & 0.006 \\
\hline
\end{tabular}


High $\mathrm{x}$ extrapolation contributes 0.003 . Low $\mathrm{x}$ extrapolation is guided by Regge form $A_{1}^{n}(\mathrm{x}) \sim \mathrm{x} \alpha$, and contributes -0.006 . Systematic uncertainties from extrapolation have been estimated by varying the range of the fit and are taken to be equal to the magnitude of the contributions. Spin Muon Collaboration (SMC) measurements at lower $\mathrm{x}$ are compatible with the E-142 extrapolation. ${ }^{23}$ Thus, the integral of $g_{1}^{n}$ over the range $\mathrm{x}=0$ to $\mathrm{x}=1$ has been determined to be $-0.022 \pm 0.007 \pm 0.009$, where the first error is statistical and the second systematic. Adding the two uncertainties in quadrature yields $\mathrm{I}^{\mathrm{n}}=-0.022 \pm 0.011$.

Table IV: Determination of the integral of $g_{1}^{n}(\mathrm{x})$ by E-142.

\begin{tabular}{|l|c|c|c|}
\hline & Integral & Statistical & Systematic \\
\hline Measured $x$ range & -0.019 & 0.007 & 0.006 \\
\hline Low $x$ extrapolation & -0.006 & & 0.006 \\
\hline High x extrapolation & 0.003 & & 0.003 \\
\hline $\mathrm{I}^{\mathrm{n}}$ & -0.022 & 0.007 & 0.009 \\
\hline
\end{tabular}

\subsection{Quark Spin and Sum Rule}

Using this value of $\mathrm{I}^{\mathrm{n}}$ in place of the EMC measurement of $\mathrm{I}$, one finds that

$$
\begin{gathered}
\Delta u=0.93 \pm 0.03, \\
\Delta d=-0.33 \pm 0.03, \\
\Delta s=0.01 \pm 0.03,
\end{gathered}
$$

$$
\Delta q=\Delta u+\Delta d+\Delta s=0.60 \pm 0.10 \text {. }
$$

In contrast to the conclusions based on IP, quarks are found to carry approximately half the spin of the nucleon, and strange sea polarization is small.

The most precise test of the Bjorken sum rule is obtained by combining this determination of $\mathrm{I}^{\mathrm{n}}$ with the results of $\mathrm{E} 143^{24}$ on the proton $\mathrm{IP}=0.127 \pm 0.004$ (stat.) \pm 0.010 (sys.), to give the experimental result $\mathrm{Ip}-\mathrm{In}^{\mathrm{n}}=0.149 \pm 0.015$. This is an agreement at the $1-\sigma$ level with the sum rule prediction of $0.164 \pm 0.008$, where we have used $\alpha_{s}=0.39 \pm 0.007$ at $\mathrm{Q}^{2}=2 \mathrm{GeV}^{2}$ to evaluate $\mathrm{QCD}$ corrections. Higher twist effects have not been included in the theoretical prediction.

\section{Other Polarized Structure Function Experiments at SLAC}

\subsection{Experiment $E-143$}

Experiment E-143 measures the polarized structure functions of the proton and deuteron with polarized $\mathrm{NH}_{3}$ and $\mathrm{ND}_{3}$ targets, respectively. Neutron results will be obtained by subtraction. Data were taken from November 1993 through February 1994. Beam parameters are similar to those for E-142, but with higher beam energy $(29 \mathrm{GeV})$ and

\footnotetext{
${ }^{23}$ See Fig. 3 in B. Adeva et al., Cem Preprint CERN-PPE/93-206.
}

${ }^{24}$ K. Abe et al., Phys. Rev. Lett. 74 (1995) 346. polarization. The use of a strained GaAs cathode 25 has led to beam polarization of $\sim 85 \%$. Results on the spin structure function of the proton ${ }^{26}$ and deuteron ${ }^{27}$ have been published.

\subsection{0-GeV Program}

An upgrade of the beam line from $30 \mathrm{GeV}$ to $50 \mathrm{GeV}$ is in progress, and two experiments have been approved to continue precision measurements of polarized nucleon structure functions. Beam polarizations of $\sim 85 \%$ are expected. E-154 will use an improved ${ }^{3} \mathrm{He}$ target, and $\mathrm{E}-155$ will use $\mathrm{NH}_{3}$ and $\mathrm{ND}_{3}$ targets. Spectrometers will be rebuilt at $2.75^{\circ}$ and $5.5^{\circ}$ to accommodate the new kinematics. Typical $\mathrm{Q}^{2}$ will increase from $\sim 2 \mathrm{GeV}^{2}(\mathrm{E}-142)$ to $\sim 5 \mathrm{GeV}^{2}$, with substantial data above $10 \mathrm{GeV}^{2}$. The lowest $\mathrm{x}$ (with $\mathrm{Q}^{2}>1 \mathrm{GeV}^{2}$ ) is reduced fom $\sim 0.035$ to $\sim 0.018$. Figure 7 shows the expected improvement to $A_{1}^{n}$. A summary of current and expected uncertainties on the integrals of structure functions is given in Table V. It is expected that the most stringent test of the Bjorken sum rule will use In from E-154 and Ip from E-155. This expected improvement is illustrated in Figs. 8 and 9 .

Table V: Measurement precision on IP, $I^{\mathrm{n}}$, and $I^{\mathrm{d}}$. The three values are statistical, systematic, and extrapolation uncertainties, respectively. E-130, E-142, and E-143 results have been published. The E-154 and E-155 numbers are from their proposals.

\begin{tabular}{|l|c|c|c|}
\hline & $\Delta \mathrm{IP}^{\mathrm{p}}$ & $\Delta \mathrm{I}^{\mathrm{n}}$ & $\Delta \mathrm{I}^{\mathrm{d}}$ \\
\hline \hline E-130 & .05 (combined) & & \\
\hline E-142 & & $.007 / .006 / .007$ & \\
\hline \hline E-143 & $.004 / .008 / .006$ & $\begin{array}{c}.008 / .011 \\
\text { (combined) }\end{array}$ & $.003 / .004 / .001$ \\
\hline E-154 & & $.003 / .003 / .003$ & \\
\hline E-155 & $.001 / .008 / .001$ & $.002 / .006 / .002$ & $.002 / .008 / .002$ \\
\hline
\end{tabular}

${ }^{25}$ T. Maruyama et al., SLAC-PUB-5731, SLAC-PUB-6033, E. Garwin et al., SLAC-PUB-5751. ${ }^{26} \mathrm{~K}$. Abe et al., Phys. Rev. Lett. 73 (1995) 346.

${ }^{27} \mathrm{~K}$. Abe et al., SLAC-PUB-6734. 


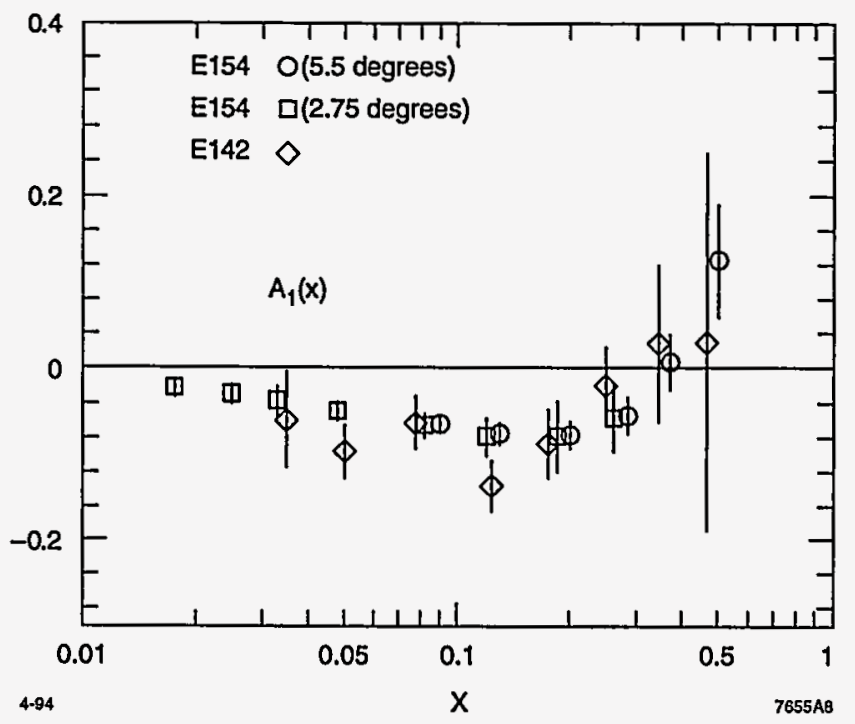

Figure 7: Expected measurement precision of $A_{1}^{n}(\mathrm{x})$ from E-154 compared with existing results.

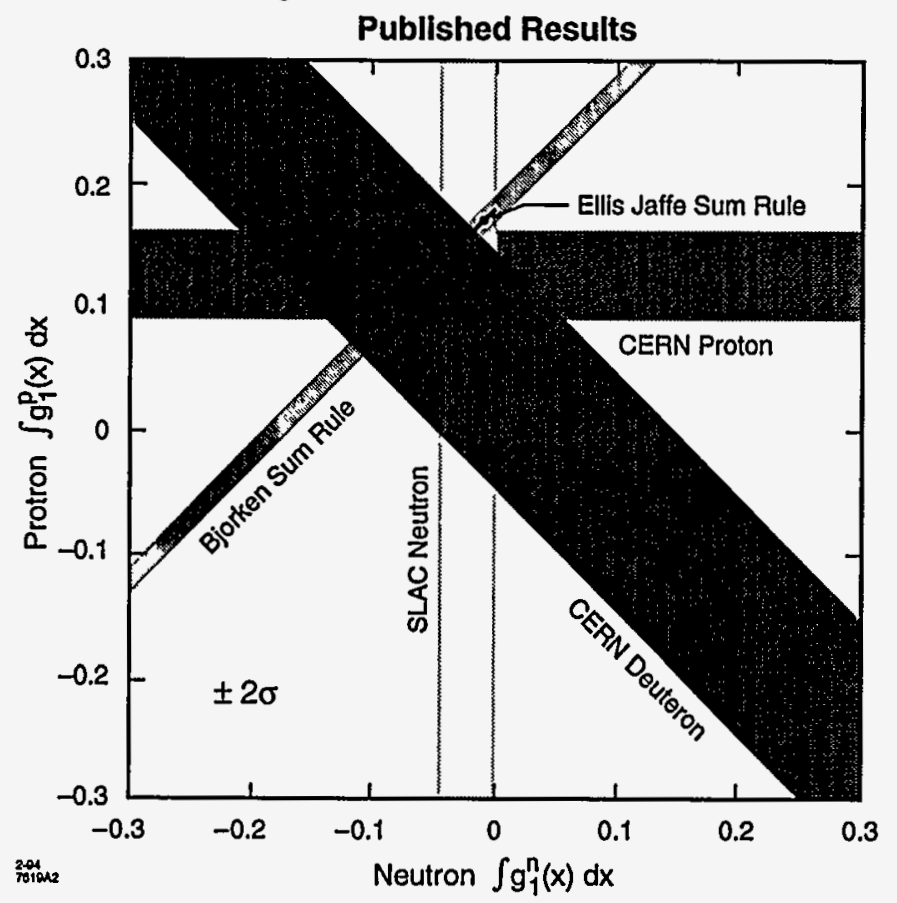

Figure 8: Precision of published results on the integrals of proton and neutron structure functions. 13

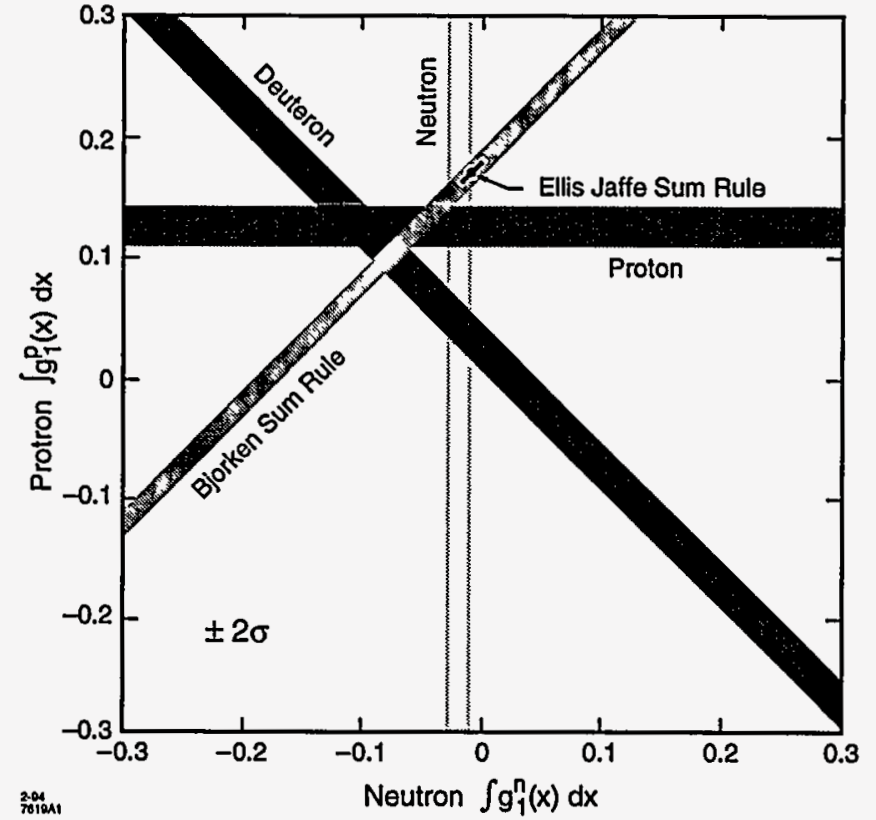

Figure 9: Expected precisions from experiments E-154 and E-155.

\section{DISCLAIMER}

This report was prepared as an account of work sponsored by an agency of the United States Government. Neither the United States Government nor any agency thereof, nor any of their employees, makes any warranty, express or implied, or assumes any legal liability or responsibility for the accuracy, completeness, or usefulness of any information, apparatus, product, or process disclosed, or represents that its use would not infringe privately owned rights. Reference herein to any specific commercial product, process, or service by trade name, trademark, manufacturer, or otherwise does not necessarily constitute or imply its endorsement, recommendation, or favoring by the United States Government or any agency thereof. The views and opinions of authors expressed herein do not necessarily state or reflect those of the United States Government or any agency thereof. 